\title{
SPECIFIC CHARACTERS USED IN THE GENUS PSEUDOCOCCUS.
}

\author{
By P. E. Sмгтн, \\ Entomological Laboratory of Cornell University.
}

INTRODUCTION.

The purpose of this investigation was to determine the value of the specific characters used in the descriptions of species of Pseudococcus. While all characters used have probably not been noted and while some of those omitted may be of considerable value still the more common and important characters have received attention.

The investigation has been limited to five species, ${ }^{*}$ namely: agrifoliae Essig, citri Risso, crawii Coq., longispinus Targ., and obscurus Essig. A large number of individuals in each species has been used giving a comparative study for specific variation.

The writer wishes to thank Professor Alex. D. MacGillivray for the many invaluable suggestions given.

\section{TABULATION AND STUDY OF CHARACTERS.}

An examination of the descriptions of species in this genus shows a great similarity in the characters used: Provided that these characters do not vary beyond certain limits, this would make the identification of the species easier. However, if these characters vary to any great extent and overlap and merge into each other, the adherence by systematists to these characters instead of the introduction of new ones is unfortunate, and would make the identification of species very difficult, if not impossible. In that case the most valuable data in the descriptions would be the locality and host-plant data.

BoDy. 1. Size.-In the great majority of descriptions, the length and width of the body is given. In the measurements given, a great variation in the length of each species is noted. $\dagger$ Lengths such as 3-4 mm., 2-5 mm., 1.5-4 mm., are very frequent, showing the wide variation noted by those describing species. The tables $f$ showing the lengths of the body, (Tables $1,2,3,4,5)$ in the five species studied do not show a variation any greater than this. A variation of from one and one-half

* The writer does not express an opinion upon the validity of these species.

$\dagger$ R. Mathewson. Can. Entom. XXXIX, p. 286.

f In these and the following tables an ocular micrometer with a 2-5 and 1-8 inch objective were used. All measurements are given in microns. 
to twice the length of the smaller specimens is shown. A greater variation would probably be found if a larger number of specimens' were' measured.

TABLE 1. Pseudococcus obscurus Essig.

\begin{tabular}{l|c|c|c|c|c}
\hline Specimen & $\begin{array}{c}\text { Length of } \\
\text { Body: }\end{array}$ & $\begin{array}{c}\text { Length of } \\
\text { Setal-lobp }\end{array}$ & \multicolumn{2}{|c|}{$\begin{array}{c}\text { Length of Setae } \\
\text { of Anal-lobes }\end{array}$} & $\begin{array}{c}\text { Length of Setae } \\
\text { of Anal-ring }\end{array}$ \\
\hline I & 2736 & 544 & 152 & 136 & 176 \\
II & & & 152 & 152 & 168 \\
III & 2784 & 160 & 160 & 184 \\
IV & 2526 & 736 & 152 & 144 & 192 \\
VI & $2304^{\circ}$ & 720 & 152 & 148 & 190 \\
IX & 3120 & $560^{\circ}$ & 156 & 146 & 185 \\
XI & 2040 & 320 & 136 & 135 & 176 \\
XII & 2688 & 848 & 137 & 145 & 168 \\
XII & 3000 & 696 & & 152 & 167 \\
IVX & 3072 & & 150 & 142 & 168 \\
\hline
\end{tabular}

TABLE 2. Pseudococcus agrifoliae Essig.

\begin{tabular}{|c|c|c|c|c|c|}
\hline Specimen & $\begin{array}{l}\text { Length of } \\
\text { Body }\end{array}$ & $\begin{array}{l}\text { Length' of } \\
\text { Setat-1oop }\end{array}$ & \multicolumn{2}{|c|}{$\begin{array}{l}\text { Length of Setie } \\
\text { of Anal-lobes }\end{array}$} & $\begin{array}{l}\text { Lerigth of Setae } \\
\text { of Anal-ting }\end{array}$ \\
\hline I & 2928: & 11.60 . & 221 & 216 & 240 \\
\hline II & 3312 & $1129^{\circ}$ & 224 & 223 & 200 \\
\hline III & 4200 & $1165^{\circ}$ & & 208 & 184 \\
\hline$V^{\prime}$ & 3336 & & 182 & 184 & 176 \\
\hline VII & 4205 & 1.20 & 268 & 225 & 292 \\
\hline $\mathrm{X}$ & 2928 & 400 & 200 & & 200 \\
\hline XIFI & 3120 & 405 & & 215 & 248 \\
\hline XVII & 4080 & & 208 & 203 & 208 \\
\hline \multicolumn{6}{|c|}{ TABEE 3 PSEUDdeoccus ciTRI RISSO. } \\
\hline Specimen: & $\begin{array}{c}\text { Length of } \\
\text { Body }\end{array}$ & $\begin{array}{l}\text { Length of } \\
\text { Setal-loop }\end{array}$ & \multicolumn{2}{|c|}{$\begin{array}{l}\text { Length of Setae } \\
\text { of Anal-lobes }\end{array}$} & $\begin{array}{c}\text { Length of Setae } \\
\text { of Anal-ring }\end{array}$ \\
\hline I & 3100 & 416 & 162 & 178 & 108 \\
\hline II & 2304 & 256 & $180^{i}$ & 163 & 108 \\
\hline IIF & 2528 & & 245 & 223: & 120 \\
\hline IV & 2808 & & 216 & 209 & 129 \\
\hline $\mathrm{V}$ & 2664 & 448 & & 228 & 118 \\
\hline VI & 2448 & 240 & 221 & 216 & 117 \\
\hline VII & 3144 & & 181 & 182 & 120 \\
\hline VİI & 2328 & & 192 & & $115^{1}$ \\
\hline IX & $2662^{\prime}$ & $144^{\circ}$ & 235 & 216 & 125 \\
\hline $\mathrm{X}$ & 2712 & & 225 & 233 & 130 \\
\hline
\end{tabular}


TABLE 4. Pseudococcus crawil Cog.

\begin{tabular}{l|c|c|c|c|c|}
\hline Specimen & $\begin{array}{c}\text { Length of } \\
\text { Body }\end{array}$ & $\begin{array}{c}\text { Length of } \\
\text { Setal-loop }\end{array}$ & \multicolumn{2}{|c|}{$\begin{array}{c}\text { Length of Setae } \\
\text { of Anal-lobes }\end{array}$} & $\begin{array}{c}\text { Length of Setae } \\
\text { of Anal-ring }\end{array}$ \\
\hline I & 3048 & 98 & 228 & 245 & 144 \\
IV & 2184 & 101 & 241 & 239 & 168 \\
V & 2160 & 120 & & 200 & 154 \\
A & 2256 & & 216 & 215 & 176 \\
B & 2160 & & & 256 & 192. \\
VI & 2165 & & & 248 & 201 \\
VII & 2208 & 108 & 208 & 201 & 169 \\
VIII & 2376 & & 200. & 176 & 160. \\
IX & 2373 & & 224 & 222 & 184 \\
X & 2448 & & 223 & 220 & 160 \\
\hline
\end{tabular}

TABLE 5. Pseudococcus longispinus Targ.

\begin{tabular}{l|c|c|c|c|c} 
Specimen & $\begin{array}{c}\text { Length of } \\
\text { Body }\end{array}$ & $\begin{array}{c}\text { Length of } \\
\text { Setal-loop }\end{array}$ & $\begin{array}{c}\text { Length of Setae } \\
\text { of Anal-lobes }\end{array}$ & $\begin{array}{c}\text { Length of Setae } \\
\text { of Anal-ring }\end{array}$ \\
\hline I & 2088 & & 132 & 120 & 139 \\
II & 2160 & & 130 & 124 & 132 \\
III & 1944 & & 96 & 108 & 120 \\
IV & 2328 & & 95 & 113 & 121 \\
VI & 2664 & & 113 & 121 & 182 \\
VII & 2592 & & 127 & 128 & 137 \\
VIII & 2952 & & 125 & 123. & 144 \\
X & 3312 & & 228 & 126 & 131 \\
A & 2950 & & 120 & 119 & 133 \\
B & 2526 & & 115 & 122 & 131 \\
\hline
\end{tabular}

Also only: the larger individuals. were studied becanse of the danger of including those in the nymphal stage. Each species was collected from the same or neighboring host plants and were apparently under similan conditions, so that size cannot be taken as a specific character of any great importance. These measurements were taken from mounts. If unmounted spec: imens: were used, the variation would probably. be still greator; as then, a second factor, namely, the amount of secretion; pres ent, would enter. Because of the greas variation in: size of the different indiriduals in a species the size of a specimen can be of but little if any value in specific determinations.

2. Shape:-In descriptions the shape of the body is vari? ously: given as elongate-owate; rourded-oxad, convex, taperigrg: at ends, elongate, etc., words which are nearly synonymous A few species are stated to be considerably oute of the ondinam 
in shape, but in general synonyms are used in the descriptions of body shape. In the species studied, this could not be an important specific character as all the species studied were of practically the same shape. However, in some species, this might be a distinguishing character.

3. Color.-In descriptions the color of specimens is usually given, being stated as, whitish, greyish, pinkish, with a red tinge; etc. The dermis of specimens in this genus is red. The color of the insect depends on the extent that this dermis is hidden by the waxy secretion. The amount of waxy secretion depends largely on the position in which the individual develops. Those in exposed position requiring more of the waxy covering as a protection than those developing in well protected situations. This variation in color is well shown in agrifoliae Essig. The color in this species is usually of a reddish, pinkish, or pinkish brown. The writer has found specimens, however, ovipositing in exposed positions which were completely covered with the waxy secretion and through which the red dermis was not visible. Thus all variations from a white to a red specimen were found. In the other species studied the color was normally white. In the five species studied color as a character for separating the species could not be relied upon.

4. Segmentation.- - In many descriptions the segmentation is referred to as distinct or not distinct. This is a character that is not used to any great extent. This is fortunate as the value of the prominence of the segmentation as a specific character is extremely doubtful. In mounted specimens it would depend to a large extent upon the method used in preparation. The segmentation in chloroform mounts is well preserved. In caustic potash mounts it would be largely obliterated. In unmounted as well as mounted specimens the age and size of the specimens is of great importance. In nymphs of Pseudococcus the segmentation is very distinct. As it passes through its last moult and becomes an adult, this distinctness of the segmentation is lost to a degree. Then as the insect becomes more and more distended with eggs this tendency to lose its prominent segmentation is increased. Also the distinctness of the segmentation in unmounted specimens would depend somewhat upon the amount of secretion covering the insect. With these factors it does not seem that the distinctness of the segmentation can be a very satisfactory specific character, 
AntennaE.-The number of segments of the antennae is a generic and not a specific character, and so is of no importance in separating species. Several species have been described with seven segmented antennae. The number of segments, eight, of the type of the genus, longispinus Targ, has been adopted in this paper. However, a dimorphism has been described in two instances, viz.: by Folsom for trifolii Forbes, and by Essig for agrifoliae Essig. In these descriptions there is said to be a winter form which has seven segmented antennae; this form giving rise to the summer form with the normal eight segmented antennae. If further investigation shows these observations to be correct, it will be of both specific and generic importance.

The comparative length of the different segments of the antennae is a character that is the most used of any of the characters of the insect's body. The relative length of the segments and the formulae deduced from the measurements is almost invariably contained in descriptions. Sometimes a considerable variation is noted, several formulae being given.

In this study ten specimens of each species were used. Aside from the question of variation which will be taken up la,ter, the relative length of the antennal segments is not a desirable character to use. The greatest difficulty in its use is the difficulty of making correct measurements and the determination of the exact limits of the various segments. The chitin is not continuous from one segment to the next and consequently the portion between the chitinous parts of the segments, the conjunctiva, is not visible or only slightly so in well cleared specimens. Consequently, in making measurements, the determination of the end of a segment will be only approximately at the center of the conjunctiva. This difficulty will be much increased if there are some bends in the antennae. A second difficulty is to determine some point at the end of the segment from which the measurement will always be taken. This difficulty is most apparent with the first segment. This segment is an irregular truncated cone with the sides of different lengths, and but little longer than the width across the base. It will be seen that the determination of the same points for the measurement of this segment would be nearly impossible. In the tables given the writer does not feel that the measurements of the first segment are dependable. Another difficulty in getting dependable formulae is the very slight difference in 
the length of some of the segments compared to each other. A difference of only two or three microns is all that is found: in some of the segments. It would be exceedingly difficult to eliminate inaccuracies to: the extent that the formulae wouldr. not be changed by them. Or in other words, the limit of erroris so small that error, even with the greatest care, is bound. to occur. Unconsciously the measurements would be made to conform to a given formala or to other measurements. The writer continually met this difficulty. The: measurements given in Tables 6-10; were taken with an ocular micnometer at a magnification: of 660 diameters. Much more accurate work was possible than would have been: with a camera lucida.

TABLE 6. PSEUdococcus crawir Coq.

\begin{tabular}{|c|c|c|c|c|c|c|c|c|c|}
\hline Specimen & list & 2 nd & $3 \mathrm{rd}$ & 4 th & 5 thr & 6th & 7 th & 8 th & Eormulae \\
\hline I & $\begin{array}{l}60 \\
65\end{array}$ & $\begin{array}{l}67 \\
67\end{array}$ & $\begin{array}{l}74 \\
77\end{array}$ & $\begin{array}{l}55 \\
53\end{array}$ & $\begin{array}{l}38 \\
48\end{array}$ & $\begin{array}{l}46 \\
41\end{array}$ & $\begin{array}{l}48 \\
48\end{array}$ & $\begin{array}{l}108^{\prime} \\
106\end{array}$ & $\begin{array}{l}88214765 \\
83214(75) 6\end{array}$ \\
\hline II & $\begin{array}{l}67 \\
62\end{array}$ & $\begin{array}{l}72 \\
79\end{array}$ & $\begin{array}{l}87 \\
84\end{array}$ & $\begin{array}{l}48 \\
46\end{array}$ & $\begin{array}{l}50 \\
48\end{array}$ & $\begin{array}{l}48 \\
46\end{array}$ & $\begin{array}{l}48 \\
53\end{array}$ & $\begin{array}{l}110 \\
1.15\end{array}$ & $\begin{array}{l}83215(647) \\
882175(64)\end{array}$ \\
\hline IrI & $\begin{array}{l}60 \\
62\end{array}$ & $\begin{array}{l}72 \\
67\end{array}$ & $\begin{array}{l}74 \\
79\end{array}$ & $\begin{array}{l}53 \\
53\end{array}$ & $\begin{array}{l}58 \\
48\end{array}$ & $\begin{array}{l}46 \\
48\end{array}$ & $\begin{array}{l}50: \\
50\end{array}$ & $\begin{array}{l}108 \\
108\end{array}$ & $\begin{array}{l}88215476 \\
832147(65)\end{array}$ \\
\hline IV & $\begin{array}{l}70 \\
70\end{array}$ & $\begin{array}{l}72: \\
77\end{array}$ & $\begin{array}{l}94 \\
96\end{array}$ & $\begin{array}{l}55 \\
46\end{array}$ & $\begin{array}{l}58 \\
58\end{array}$ & $\begin{array}{l}53 \\
48\end{array}$ & $\begin{array}{l}48 \\
48\end{array}$ & $\begin{array}{l}108 \\
118\end{array}$ & $\begin{array}{l}83215467 \\
88215(67 .) 4\end{array}$ \\
\hline $\mathrm{V}$ & $\begin{array}{l}60 \\
67\end{array}$ & $\begin{array}{l}72 \\
74\end{array}$ & $\begin{array}{l}96 \\
94\end{array}$ & $\begin{array}{l}50 \\
43\end{array}$ & $\begin{array}{l}60 \\
58\end{array}$ & $\begin{array}{l}53 \\
48\end{array}$ & $\begin{array}{l}48 \\
50\end{array}$ & $\begin{array}{l}1115: \\
1117\end{array}$ & $\begin{array}{l}83215647 \\
8921 \times 57.04\end{array}$ \\
\hline VI & $\begin{array}{l}62 \\
65\end{array}$ & $\begin{array}{l}77 \\
79\end{array}$ & $\begin{array}{l}91 \\
86\end{array}$ & $\begin{array}{l}46 \\
48\end{array}$ & $\begin{array}{l}55 \\
70\end{array}$ & $\begin{array}{l}50 \\
46\end{array}$ & $\begin{array}{l}50 \\
48\end{array}$ & $\begin{array}{l}1: 18 \\
\text { lito }\end{array}$ & $\begin{array}{l}83215(67) 4 \\
89251(47) 6\end{array}$ \\
\hline VIII & $\begin{array}{l}62 \\
62\end{array}$ & $\begin{array}{l}70 \\
87\end{array}$ & $\begin{array}{l}82 \\
82\end{array}$ & $\begin{array}{l}53 \\
50\end{array}$ & $\begin{array}{l}53 \\
53\end{array}$ & $\begin{array}{l}53 \\
50\end{array}$ & $\begin{array}{l}46: \\
50\end{array}$ & $\begin{array}{l}115 \\
1115\end{array}$ & $\begin{array}{l}88221(456) 7 \\
88215(467)\end{array}$ \\
\hline IX & $\begin{array}{l}60 \\
60 .\end{array}$ & $\begin{array}{l}67 \\
70\end{array}$ & $\begin{array}{l}74 \\
7.4\end{array}$ & $\begin{array}{l}48 \\
48\end{array}$ & $\begin{array}{l}55 \\
65\end{array}$ & $\begin{array}{l}48 \\
48\end{array}$ & $\begin{array}{l}53 \\
53 .\end{array}$ & $\begin{array}{l}108 \\
113\end{array}$ & $\begin{array}{l}832157(64) \\
832457(64)\end{array}$ \\
\hline $\mathrm{X}$ & $\begin{array}{l}60 \\
58\end{array}$ & $\begin{array}{l}67 \\
67\end{array}$ & $\begin{array}{l}79 \\
77 .\end{array}$ & $\begin{array}{l}48 \\
48\end{array}$ & $\begin{array}{l}50 \\
52\end{array}$ & $\begin{array}{l}46 \\
43\end{array}$ & $\begin{array}{l}50 \\
48\end{array}$ & $\begin{array}{l}108 \\
108\end{array}$ & $\begin{array}{l}8321(57) 46 \\
83215(47) 6\end{array}$ \\
\hline $\mathrm{XI}$ & $\begin{array}{l}65 \\
60\end{array}$ & $\begin{array}{l}67 \\
69\end{array}$ & $\begin{array}{l}72 \\
77\end{array}$ & $\begin{array}{l}48 \\
43\end{array}$ & $\begin{array}{l}48 \\
48\end{array}$ & $\begin{array}{l}53 ! \\
41\end{array}$ & $\begin{array}{l}50 \\
48\end{array}$ & $\begin{array}{l}106 \\
106\end{array}$ & $\begin{array}{l}832167(45) \\
8321(57) 46\end{array}$ \\
\hline
\end{tabular}

An examination of the tables: mentioned will show the great variation met with. Following herewith is a disetussion of the tables of each species:-

Crawn Coq. Formula $(2,3,8), 5,4,7,6$, 1, Coq. West. A'm. Scientist. ' 89. 
There is no question about the determination of this species. They were all taken from one plant of white sage (Ramona poly'stachia) at Santa Paula, California. In the tables a formula is found which agrees with the one given by Coquillett. In but one specimen was the formula the same for the right and left antennae of the same individual. All the other formulae differed as much as the specifically diagnostic formulae published for all the species of Pseudococcus.

Lon.gispinus TARg. Formula, $(2,3,8)(1,4,5,6) 7$. Newstead, "British Coccidae." Vol. II. The specimens examined were taken from palms in the Horticultural Forcing-house of Cornell University. Of the ter specimens measured, the formulae of the right and left antennae of but one specimen were identical: No formula was found which agreed with the one given by Newstead. The formulae varied as much as the specifically diagnostic formulae published for all the species of Pseudococcus.

TABLE 7. PsEUdoCocCuS longIsPINUS. TARG.

\begin{tabular}{|c|c|c|c|c|c|c|c|c|c|}
\hline Specimen & Ist & $2 \mathrm{nd}$ & $3 \mathrm{rd}$ & 4th & 5 th & 6 th & 7 th & 8 th & Formulae \\
\hline I & $\begin{array}{l}79 \\
84\end{array}$ & $\begin{array}{l}79 \\
79\end{array}$ & $\begin{array}{l}84 \\
84\end{array}$ & $\begin{array}{l}41 \\
43\end{array}$ & $\begin{array}{l}53 \\
50\end{array}$ & $\begin{array}{l}43 \\
43\end{array}$ & $\begin{array}{l}50 \\
48\end{array}$ & $\begin{array}{r}101 \\
96\end{array}$ & $\begin{array}{l}83(12) 5746 \\
8(31) 257(64)\end{array}$ \\
\hline $1 \mathrm{I}$ & $\begin{array}{l}65 \\
67\end{array}$ & $\begin{array}{l}72 \\
67\end{array}$ & $\begin{array}{l}79 \\
74\end{array}$ & $\begin{array}{l}36 \\
38\end{array}$ & $\begin{array}{l}41 \\
46\end{array}$ & $\begin{array}{l}38 \\
36\end{array}$ & $\begin{array}{l}46 \\
43\end{array}$ & $\begin{array}{l}103 \\
101\end{array}$ & $\begin{array}{l}83217564 \\
83(12) 5746\end{array}$ \\
\hline III & $\begin{array}{l}58 \\
60\end{array}$ & $\begin{array}{l}65 \\
62\end{array}$ & $\begin{array}{l}63 \\
60\end{array}$ & $\begin{array}{l}36 \\
36\end{array}$ & $\begin{array}{l}41 \\
43\end{array}$ & $\begin{array}{l}38 \\
34\end{array}$ & $\begin{array}{l}43 \\
41\end{array}$ & $\begin{array}{l}96 \\
91\end{array}$ & $\begin{array}{l}88317564 \\
82(13) 5746\end{array}$ \\
\hline IV & $\begin{array}{l}62 \\
58\end{array}$ & $\begin{array}{l}68 \\
58\end{array}$ & $\begin{array}{l}70 \\
70\end{array}$ & $\begin{array}{l}31 \\
34\end{array}$ & $\begin{array}{l}48 \\
48\end{array}$ & $\begin{array}{l}41 \\
38\end{array}$ & $\begin{array}{l}48 \\
43\end{array}$ & $\begin{array}{r}108 \\
98\end{array}$ & $\begin{array}{l}83(21)(75) 64 \\
83(21) 5764\end{array}$ \\
\hline V & $\begin{array}{l}55 \\
58\end{array}$ & $\begin{array}{l}72 \\
72\end{array}$ & $\begin{array}{l}67 \\
6 \bar{b}\end{array}$ & $\begin{array}{l}29 \\
36\end{array}$ & $\begin{array}{l}50 \\
48\end{array}$ & $\begin{array}{l}29 \\
43\end{array}$ & $\begin{array}{l}43 \\
46\end{array}$ & $\begin{array}{l}106 \\
106\end{array}$ & $\begin{array}{l}823157(46) \\
82315764\end{array}$ \\
\hline VI & $\begin{array}{l}62 \\
60\end{array}$ & 72 & $\begin{array}{l}74 \\
74\end{array}$ & $\begin{array}{l}43 \\
46\end{array}$ & $\begin{array}{l}60 \\
58\end{array}$ & $\begin{array}{l}46 \\
48\end{array}$ & $\begin{array}{l}48 \\
48\end{array}$ & $\begin{array}{l}106 \\
106\end{array}$ & $\begin{array}{l}83215764 \\
82315(76) 4\end{array}$ \\
\hline VII & $\begin{array}{l}74 \\
72\end{array}$ & $\begin{array}{l}72 \\
82\end{array}$ & $\begin{array}{l}74 \\
91\end{array}$ & $\begin{array}{l}48 \\
50\end{array}$ & $\begin{array}{l}62 \\
62\end{array}$ & $\begin{array}{l}43 \\
46\end{array}$ & $\begin{array}{l}43: \\
48\end{array}$ & $\begin{array}{l}101 \\
103\end{array}$ & $\begin{array}{l}8(13) 254(67) \\
88215476\end{array}$ \\
\hline VIII & $\begin{array}{l}65 \\
70\end{array}$ & $\begin{array}{l}67 \\
72\end{array}$ & $\begin{array}{l}70 \\
70\end{array}$ & $\begin{array}{l}43 \\
41\end{array}$ & $\begin{array}{l}50 \\
46\end{array}$ & $\begin{array}{l}41 \\
43\end{array}$ & $\begin{array}{l}4 B \\
46\end{array}$ & $\begin{array}{l}98 \\
96\end{array}$ & $\begin{array}{l}8321.5(47) 6 \\
82(31)(75) 64\end{array}$ \\
\hline IX & $\begin{array}{l}55 \\
60\end{array}$ & $\begin{array}{l}65 \\
65\end{array}$ & $\begin{array}{l}65 \\
65\end{array}$ & $\begin{array}{l}36 \\
34\end{array}$ & $\begin{array}{l}48 \\
50\end{array}$ & $\begin{array}{l}38 \\
41\end{array}$ & $\begin{array}{l}46 \\
43\end{array}$ & $\begin{array}{l}101 \\
+101\end{array}$ & $\begin{array}{l}8(32) 15764 \\
8(23) 15764\end{array}$ \\
\hline $\mathrm{X}$ & $\begin{array}{l}62 \\
62\end{array}$ & $\begin{array}{l}72 \\
70\end{array}$ & $\begin{array}{l}65 \\
65\end{array}$ & $\begin{array}{l}43 \\
41\end{array}$ & $\begin{array}{l}58 \\
48\end{array}$ & $\begin{array}{l}41 \\
43\end{array}$ & $\begin{array}{l}48 \\
43\end{array}$ & $\begin{array}{l}101 \\
103\end{array}$ & $\begin{array}{l}82315746 \\
82315(76) 4\end{array}$ \\
\hline
\end{tabular}


TABLE. 8. Pseudococcus agrifoliae Essig.

\begin{tabular}{|c|c|c|c|c|c|c|c|c|c|}
\hline Specimen & $1 \mathrm{st}$ & 2nd & $3 \mathrm{rd}$ & 4 th & 5 th & 6 thi & 7th & 8 th & Formulae \\
\hline I & $\begin{array}{l}94 \\
89\end{array}$ & $\begin{array}{l}77 \\
82\end{array}$ & $\begin{array}{l}74 \\
74\end{array}$ & $\begin{array}{l}50 \\
53\end{array}$ & $\begin{array}{l}55 \\
55\end{array}$ & $\begin{array}{l}50 \\
53\end{array}$ & $\begin{array}{l}48 \\
48\end{array}$ & $\begin{array}{l}108 \\
115\end{array}$ & $\begin{array}{l}81235(46) 7 \\
81235(46) 7\end{array}$ \\
\hline II & 72 & $\begin{array}{l}72 \\
74\end{array}$ & $\begin{array}{l}74 \\
72\end{array}$ & $\begin{array}{l}48 \\
48\end{array}$ & $\begin{array}{l}58 \\
48\end{array}$ & $\begin{array}{l}48 \\
53\end{array}$ & $\begin{array}{r}41 \\
-53 \\
\end{array}$ & $\begin{array}{l}106 \\
108\end{array}$ & $\begin{array}{c}83(21) 5(46) 7 \\
\end{array}$ \\
\hline V & $\begin{array}{l}72 \\
72\end{array}$ & $\begin{array}{r}79 \\
.79\end{array}$ & $\begin{array}{l}72 \\
72\end{array}$ & $\begin{array}{l}48 . \\
48 .\end{array}$ & $\begin{array}{l}48 \\
53\end{array}$ & $\begin{array}{l}48 \\
53\end{array}$ & $\begin{array}{l}50 \\
.43\end{array}$ & $\begin{array}{l}113 \\
110\end{array}$ & $\begin{array}{l}82(13) 7(456) \\
82(13)(56) 47\end{array}$ \\
\hline VII & $\begin{array}{l}72 \\
72\end{array}$ & $\begin{array}{l}82 \\
82\end{array}$ & $\begin{array}{l}72 \\
70\end{array}$ & $\begin{array}{l}53 \\
53\end{array}$ & $\begin{array}{l}60 \\
60\end{array}$ & $\begin{array}{l}50 \\
50\end{array}$ & $\begin{array}{l}48 \\
53\end{array}$ & $\begin{array}{l}115 \\
118\end{array}$ & $\begin{array}{l}82(13) 5467 \\
82135(47) 6\end{array}$ \\
\hline VHII & $\begin{array}{l}74 \\
72\end{array}$ & $\begin{array}{l}72 \\
70\end{array}$ & $\begin{array}{l}74 \\
79\end{array}$ & $\begin{array}{l}89 \\
89\end{array}$ & $\begin{array}{l}48 \\
48\end{array}$ & $\begin{array}{l}53 \\
98\end{array}$ & $\begin{array}{r}108 \\
98\end{array}$ & & $\begin{array}{l}74(13) 265 \\
(76) 43125\end{array}$ \\
\hline XIII & $\begin{array}{l}74 \\
77\end{array}$ & $\begin{array}{l}94 \\
84\end{array}$ & $\begin{array}{l}82 \\
82\end{array}$ & $\begin{array}{l}65 \\
65\end{array}$ & $\begin{array}{r}70 \\
72\end{array}$ & $\begin{array}{l}55 \\
60\end{array}$ & $\begin{array}{l}48 \\
53\end{array}$ & $\begin{array}{l}113 \\
115\end{array}$ & $\begin{array}{l}82315467 \\
82315467\end{array}$ \\
\hline XIV & $\begin{array}{l}82 \\
79\end{array}$ & $\begin{array}{l}82 \\
79\end{array}$ & $\begin{array}{l}74 \\
77\end{array}$ & $\begin{array}{l}53 \\
50\end{array}$ & $\begin{array}{l}60 \\
65\end{array}$ & $\begin{array}{l}53 \\
50\end{array}$ & $\begin{array}{l}55 \\
50\end{array}$ & $\begin{array}{l}110 \\
120\end{array}$ & $\begin{array}{l}8(12) 357(46) \\
8(12) 35(467)\end{array}$ \\
\hline XVI & $\begin{array}{l}77 \\
74\end{array}$ & $\begin{array}{l}84 \\
79\end{array}$ & $\begin{array}{l}74 \\
74\end{array}$ & $\begin{array}{l}55 \\
50\end{array}$ & $\begin{array}{l}60 \\
62\end{array}$ & $\begin{array}{l}50 \\
58\end{array}$ & $\begin{array}{l}53 \\
50\end{array}$ & $\begin{array}{l}115 \\
110\end{array}$ & $\begin{array}{l}82135476 \\
82(13) 56(47)\end{array}$ \\
\hline XVII & $\begin{array}{l}72 \\
72\end{array}$ & $\begin{array}{l}77 \\
82\end{array}$ & $\begin{array}{l}72 \\
74\end{array}$ & $\begin{array}{l}53 \\
55\end{array}$ & $\begin{array}{l}60 \\
55\end{array}$ & $\begin{array}{l}53 \\
53\end{array}$ & $\begin{array}{l}50 \\
46\end{array}$ & $\begin{array}{l}115 \\
110\end{array}$ & $\begin{array}{l}82(13) 5(46) 7 \\
8231(45) 67\end{array}$ \\
\hline
\end{tabular}

Agrifoliae Essig. No formula is given in the description. The description refers however to the figures of the antennae for the relative length of the segments. Measuring the figure, the following formula is constructed, $7,1,3,2,4,6,5$. The figure is evidently taken from a nymph, as this species has normally eight segmented antennae. These specimens were all taken from a single oak tree (Quercus agrifoliae) at Santa Paula, California, and are from the lot of specimens that the type of the species was taken. In two specimens the formulae of the right and left antennae were found to be the same although the formula of each specimen is different. The formulae of the twenty antennae varied as much as the specifically diagnostic formulae published for the species of Pseudocoecus. 
TABLE 9. Pseudococcus citri Risso.

\begin{tabular}{|c|c|c|c|c|c|c|c|c|c|}
\hline Specimen & $1 \mathrm{st}$ & 2nd & $3 \mathrm{rd}$ & 4th & 5 th & 6 th & 7th & Sth & Formulae \\
\hline I & $\begin{array}{l}67 \\
62\end{array}$ & $\begin{array}{l}60 \\
60\end{array}$ & $\begin{array}{l}58 \\
60\end{array}$ & $\begin{array}{l}41 \\
43\end{array}$ & $\begin{array}{l}41 \\
43\end{array}$ & $\begin{array}{l}41 \\
48\end{array}$ & $\begin{array}{l}43 \\
48\end{array}$ & $\begin{array}{l}101 \\
103\end{array}$ & $\begin{array}{l}81237(456) \\
81(23)(67)(45)\end{array}$ \\
\hline II & $\begin{array}{l}55 \\
60\end{array}$ & $\begin{array}{l}60 \\
58\end{array}$ & $\begin{array}{l}55 \\
50\end{array}$ & $\begin{array}{l}38 \\
36\end{array}$ & $\begin{array}{l}36 \\
41\end{array}$ & $\begin{array}{l}46 \\
38\end{array}$ & $\begin{array}{l}46 \\
43\end{array}$ & $\begin{array}{l}103 \\
101\end{array}$ & $\begin{array}{l}82(13)(67) 45 \\
81237564\end{array}$ \\
\hline II I & $\begin{array}{l}60 \\
60\end{array}$ & $\begin{array}{l}67 \\
6 j\end{array}$ & $\begin{array}{l}72 \\
72\end{array}$ & $\begin{array}{l}41 \\
41\end{array}$ & $\begin{array}{l}48 \\
48\end{array}$ & $\begin{array}{l}46 \\
46\end{array}$ & $\begin{array}{l}55 \\
53\end{array}$ & $\begin{array}{l}106 \\
108\end{array}$ & $\begin{array}{l}83217564 \\
83217564\end{array}$ \\
\hline IV & $\begin{array}{l}60 \\
65\end{array}$ & $\begin{array}{l}65 \\
60\end{array}$ & $\begin{array}{l}60 \\
62\end{array}$ & $\begin{array}{l}36 \\
36\end{array}$ & $\begin{array}{l}41 \\
36\end{array}$ & $\begin{array}{l}36 \\
38\end{array}$ & $\begin{array}{l}50 \\
48\end{array}$ & $\begin{array}{l}110 \\
108\end{array}$ & $\begin{array}{l}82(13) 75(64) \\
813276(54)\end{array}$ \\
\hline$V$ & $\begin{array}{l}60 \\
58\end{array}$ & $\begin{array}{l}62 \\
62\end{array}$ & $\begin{array}{l}67 \\
65\end{array}$ & $\begin{array}{l}36 \\
36\end{array}$ & $\begin{array}{l}48 \\
43\end{array}$ & $\begin{array}{l}43 \\
43\end{array}$ & $\begin{array}{l}53 \\
50\end{array}$ & $\begin{array}{l}113 \\
113\end{array}$ & $\begin{array}{l}83217564 \\
83217(65) 4\end{array}$ \\
\hline VI & $\begin{array}{l}65 \\
62\end{array}$ & $\begin{array}{l}72 \\
72\end{array}$ & $\begin{array}{l}67 \\
65\end{array}$ & $\begin{array}{l}46 \\
48\end{array}$ & $\begin{array}{l}48 \\
46\end{array}$ & $\begin{array}{l}48 \\
50\end{array}$ & $\begin{array}{l}50 \\
53\end{array}$ & $\begin{array}{l}103 \\
110\end{array}$ & $\begin{array}{l}82317(56) 4 \\
82317645\end{array}$ \\
\hline VII & $\begin{array}{l}67 \\
67\end{array}$ & $\begin{array}{l}60 \\
58\end{array}$ & $\begin{array}{l}67 \\
60\end{array}$ & $\begin{array}{l}36 \\
38\end{array}$ & $\begin{array}{l}38 \\
41\end{array}$ & $\begin{array}{l}41 \\
43\end{array}$ & $\begin{array}{l}48 \\
43\end{array}$ & $\begin{array}{r}106 \\
98\end{array}$ & $\begin{array}{l}8(13) 27654 \\
8132(67) 54\end{array}$ \\
\hline VIII & $\begin{array}{l}67 \\
72\end{array}$ & $\begin{array}{l}72 \\
62\end{array}$ & $\begin{array}{l}67 \\
67\end{array}$ & $\begin{array}{l}38 \\
41\end{array}$ & $\begin{array}{l}43 \\
48\end{array}$ & $\begin{array}{l}43 \\
43\end{array}$ & $\begin{array}{l}46 \\
48\end{array}$ & $\begin{array}{l}108 \\
103\end{array}$ & $\begin{array}{l}82(13) 7(65) 4 \\
8132(75) 64\end{array}$ \\
\hline IX & $\begin{array}{l}60 \\
60\end{array}$ & $\begin{array}{l}67 \\
65\end{array}$ & $\begin{array}{l}60 \\
60\end{array}$ & $\begin{array}{l}43 \\
43\end{array}$ & $\begin{array}{l}43 \\
41\end{array}$ & $\begin{array}{l}38 \\
46\end{array}$ & $\begin{array}{l}41 \\
43\end{array}$ & $\begin{array}{l}96 \\
96\end{array}$ & $\begin{array}{l}82(13)(45) 76 \\
82(13) 6(74) 5\end{array}$ \\
\hline $\mathrm{X}$ & $\begin{array}{l}67 \\
67\end{array}$ & $\begin{array}{l}67 \\
65\end{array}$ & $\begin{array}{l}72 \\
67\end{array}$ & $\begin{array}{l}41 \\
43\end{array}$ & $\begin{array}{l}41 \\
48\end{array}$ & $\begin{array}{l}41 \\
46\end{array}$ & $\begin{array}{l}50 \\
48\end{array}$ & $\begin{array}{l}108 \\
108\end{array}$ & $\begin{array}{l}83(12) 7(456) \\
8(31) 2(75) 64\end{array}$ \\
\hline
\end{tabular}

Citri Risso. Formula 6, 3, 2, 1, 5, (4, 6, 7) Newstead, "British Coccidae" Vol. II. The specimens of this species were taken from coleus in the Horticultural Forcing-houses of Cornell University. No formula was found that agreed with the one given by Newstead. In one specimen the formulae for the right and left antennae were identical. The formulae of the twenty antennae varied as much as the specifically diagnostic formulae published for all the species of Pseudococcus.

Obscurus Essig. Formula 8, 1, 3, 2, 4, 7, 5, 6. Essig, "Pomona Jour. Ent." '09. The specimens of this species were taken from an elder tree (Sambucus glauca) at Santa Paula, California, and are from the lot of specimens that the type for the species was taken. In one specimen the formulae of the right and left antennae were identical. 
TABLE 10. Pseudococcus obscurus Essig.

\begin{tabular}{|c|c|c|c|c|c|c|c|c|c|}
\hline Specimen & 1st & 2nd & $3 \mathrm{rd}$ & 4 th & 5 th & 6 th & $7 \mathrm{th}$ & 8th & Formulae \\
\hline I & $\begin{array}{l}84 \\
65\end{array}$ & $\begin{array}{l}72 \\
67\end{array}$ & $\begin{array}{l}77 \\
65\end{array}$ & $\begin{array}{l}41 \\
53\end{array}$ & $\begin{array}{l}58 \\
58\end{array}$ & $\begin{array}{l}41 \\
96\end{array}$ & 53 & 103 & $\begin{array}{l}813257(64) \\
62(31) 54\end{array}$ \\
\hline II & $\begin{array}{l}84 \\
72\end{array}$ & $\begin{array}{l}72 \\
75\end{array}$ & $\begin{array}{l}79 \\
84\end{array}$ & $\begin{array}{l}48 \\
43\end{array}$ & $\begin{array}{l}55 \\
53\end{array}$ & $\begin{array}{l}46 \\
43\end{array}$ & $\begin{array}{l}48 \\
48\end{array}$ & $\begin{array}{l}84 \\
98\end{array}$ & $\begin{array}{l}(81) 325(47) 6 \\
832157(64)\end{array}$ \\
\hline III & $\begin{array}{l}82 \\
72\end{array}$ & $\begin{array}{l}79 \\
79\end{array}$ & $\begin{array}{l}84 \\
79\end{array}$ & $\begin{array}{l}29 \\
38\end{array}$ & $\begin{array}{l}60 \\
53\end{array}$ & $\begin{array}{l}50 \\
46\end{array}$ & $\begin{array}{l}53 \\
50\end{array}$ & $\begin{array}{l}101 \\
103\end{array}$ & $\begin{array}{l}83125764 \\
8(32) 15764\end{array}$ \\
\hline IV & $\begin{array}{l}82 \\
77\end{array}$ & $\begin{array}{l}89 \\
79\end{array}$ & $\begin{array}{l}84 \\
86\end{array}$ & $\begin{array}{l}48 \\
48\end{array}$ & $\begin{array}{l}65 \\
65\end{array}$ & $\begin{array}{l}50 \\
48\end{array}$ & $\begin{array}{l}50 \\
48\end{array}$ & $\begin{array}{l}108 \\
108\end{array}$ & $\begin{array}{l}82315(67) 4 \\
83215(764)\end{array}$ \\
\hline $\mathrm{V}$ & $\begin{array}{l}89 \\
74\end{array}$ & $\begin{array}{l}86 \\
84\end{array}$ & $\begin{array}{l}91 \\
96\end{array}$ & $\begin{array}{l}53 \\
50\end{array}$ & $\begin{array}{l}58 \\
65\end{array}$ & $\begin{array}{l}48 \\
55\end{array}$ & $\begin{array}{l}50 \\
48\end{array}$ & $\begin{array}{l}113 \\
113\end{array}$ & $\begin{array}{l}\$ 3125476 \\
83215647\end{array}$ \\
\hline VI & $\begin{array}{l}79 \\
74\end{array}$ & $\begin{array}{l}74 \\
72\end{array}$ & $\begin{array}{l}82 \\
82\end{array}$ & $\begin{array}{l}38 \\
36\end{array}$ & $\begin{array}{l}65 \\
60\end{array}$ & $\begin{array}{l}41 \\
41\end{array}$ & $\begin{array}{l}50 \\
48\end{array}$ & $\begin{array}{r}106 \\
98\end{array}$ & $\begin{array}{l}83125764 \\
83125764\end{array}$ \\
\hline VII & $\begin{array}{l}77 \\
89\end{array}$ & $\begin{array}{l}82 \\
74\end{array}$ & $\begin{array}{l}84 \\
74\end{array}$ & $\begin{array}{l}38 \\
41\end{array}$ & $\begin{array}{l}53 \\
53\end{array}$ & $\begin{array}{l}41 \\
41\end{array}$ & $\begin{array}{l}50 \\
46\end{array}$ & $\begin{array}{l}108 \\
106\end{array}$ & $\begin{array}{l}83215764 \\
81(23) 57(64)\end{array}$ \\
\hline VIII & $\begin{array}{l}84 \\
84\end{array}$ & $\begin{array}{l}89 \\
84\end{array}$ & $\begin{array}{l}89 \\
96\end{array}$ & $\begin{array}{l}53 \\
48\end{array}$ & $\begin{array}{l}67 \\
70\end{array}$ & $\begin{array}{l}48 \\
41\end{array}$ & $\begin{array}{l}53 \\
50\end{array}$ & $\begin{array}{l}108 \\
110\end{array}$ & $\begin{array}{l}8(32) 15(74) 6 \\
\$ 3(21) 5746\end{array}$ \\
\hline IX & $\begin{array}{l}72 \\
65\end{array}$ & $\begin{array}{l}79 \\
65\end{array}$ & $\begin{array}{l}74 \\
60\end{array}$ & $\begin{array}{l}41 \\
46\end{array}$ & $\begin{array}{l}60 \\
53\end{array}$ & $\begin{array}{r}48 \\
146\end{array}$ & 53 & 106 & $\begin{array}{l}82315764 \\
6(12) 354\end{array}$ \\
\hline $\mathrm{X}$ & $\begin{array}{l}84 \\
86\end{array}$ & $\begin{array}{l}72 \\
74\end{array}$ & $\begin{array}{l}84 \\
84\end{array}$ & $\begin{array}{l}48 \\
46\end{array}$ & $\begin{array}{l}58 \\
60\end{array}$ & $\begin{array}{l}46 \\
34\end{array}$ & $\begin{array}{l}55 \\
60\end{array}$ & $\begin{array}{c}55 ; 43 \\
108\end{array}$ & $\begin{array}{l}(13) 25(78) 469 \\
8132(75) 46\end{array}$ \\
\hline
\end{tabular}

No formula was found agreeing with the one quoted. In specimen $\mathrm{X}$ the right antenna has nine segments and in specimen IX the left antenna has six segments. An examination of Table 1 shows that this specimen is the largest specimen studied, and as it was found in an egg mass it was undoubtedly an adult. Specimen I, which will be seen to be of normal size (Table 1), also had the left antenna with six segments. This variation in the number of segments was also noted in other specimens. The formulae of the twenty antennae measured varied as much as the specifically diagnostic formulae for all the species of Pseudococcus, as well as one formula placing the specimen in the genus Phenacoccus and two formulae placing the specimens in the genus Ripersia.

From the above review it will be readily seen that the relative length of the segments of the antennae are vaiueless as specific characters. Other workers as well have found this 
character very variable. Kellogg \& Smith, '04, found that in twenty-five specimens of Cereputo yuccae, a closely allied genus, no two formulae agreed, "and that there was practically as much variety in these formulae as there is among the eleven formulae published as specifically diagnostic for eleven North American specics of the genera Cereputo and Phenacoccus." Again Tinsley, J. D., ' 08 , in discussing the variation in the antennae of $P$. virgatus Ckll. gives eleven different formulae. Again the same author, 1900, in his description of a new species, $P$. texensis Tins., gives three different formulae. Ehrhorn, Edw. M., 1900, in his description of a new species, $P$. maritimus Ehr., gives four formulae. The universal use of antennal formulae in descriptions is unfortunate, as this has given an excuse for the creation of new species and is valueless in analytical tables for the determination of specimens. The sooner that the valuelessness of this character is realized by systematists describing new species of this genus, the sooner will a search for valuable characters be begun and a serious mistake in taxonomy be corrected.

Legs. Tables 11-15. The length of the legs is used to quite an extent in descriptions. Their length compared to the length of the antennae is often stated, also the length of the different segments compared with each other. They are often spoken of as long and strong. The presence of hairs is often noted, also that of knobbed digitules.

For the study of the legs five specimens of each species were used. They were the same specimens that were used in the study of the antennae. The measurements of the different segments are the greatest length of these segments, so that the sum of the lengths of the different segments will be greater than the length of the leg. Like the basal segment of the antennae, the coxa is very difficult to measure and the results are not entirely reliable.

At the right hand side of the tables, formulae are appended. These formulae are constructed in the same manner as the antennal formulae. The segments are numbered in order beginning with the coxa.

The formulae show but little if any more satisfactory results for specific determination than do the formulae of the antennae. The formulae of each pair of legs for each of the species will be discussed together. 
Prothoracic Legs. In crawii Coq. and citri Risso the third segment is always the longest, the fourth segment coming next in order. In the remaining three species the third segment is usually the longest but may be equal to or less than the fourth segment. The first segment is always third in order in the formulae. In no species does the second segment bear any fixed relation to the fifth. The sixth segment is always much the shortest and comes last in order in the formulae.

Mesothoracic Legs. In crawii Coq. the third segment is always the longest. In the other species the position of the third and fourth segments vary in relation to each other. The first segment always comes third in the formulae. The second and fifth segments vary in relation to each other in each of the species. The sixth segment always comes last in the formulae.

Metathoracic Legs. In obscurus Essig and citri Risso the fourth segment is usually longer than the third. In obscurus Essig an exception is seen to this in the right leg of Specimen XI. In obscurus Risso an exception is seen to this in specimen VII. In the other species the fourth segment is always longer than the third. The first segment always comes third in the formulae. In no species do the second and third segments bear any fixed relation to each other. The sixth segment is always much the shortest and comes last.in the formulae.

It will be seen from the above discussion that the variation in the formulae is too great for them to be of service in specific determination. Exceptions are found to any generalization that might be made. The limits within which there can be variation are so small that the variations are almost sure to go beyond these limits. Other parts of the legs as setae, digitules, etc., apparently offer no characters of a specific nature. 
TABLE 11. Pseudococcus crawir CoQ.

\begin{tabular}{|c|c|c|c|c|c|c|c|c|c|}
\hline $\begin{array}{l}\text { Seg- } \\
\text { ment }\end{array}$ & $\begin{array}{l}\text { Spec- } \\
\text { imen }\end{array}$ & Side & Coxa & Tr. & $\begin{array}{l}\text { Fe- } \\
\text { mur }\end{array}$ & Tibia & $\begin{array}{l}\text { Tar- } \\
\text { sus }\end{array}$ & Claws & Formulae \\
\hline \multirow{9}{*}{ 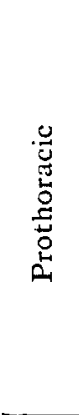 } & I & Rt. & $\begin{array}{l}192 \\
198\end{array}$ & 120 & 288 & 252 & 114 & $\begin{array}{l}39 \\
36\end{array}$ & 341256 \\
\hline & TU & & & & & & & & \\
\hline & 17 & Kf. & $\begin{array}{l}240 \\
228\end{array}$ & 126 & $\begin{array}{l}312 \\
318\end{array}$ & $\begin{array}{l}248 \\
276\end{array}$ & 120 & $\begin{array}{l}42 \\
42\end{array}$ & $\begin{array}{l}341256 \\
341(25) 6\end{array}$ \\
\hline & $Y$ & & & & & 240 & 108 & & 241256 \\
\hline & & Lft. & 222 & 120 & 270 & 240 & 108 & 42 & 341256 \\
\hline & VIII & Rt. & 210 & 120 & 276 & 216 & 108 & 30 & 341256 \\
\hline & & Lft. & 210 & 120 & 282 & 216 & 108 & 30 & 341256 \\
\hline & IX & Rt. & & & & & & & \\
\hline & & Lft. & 204 & 114 & 288 & 252 & 114 & 36 & $341(25) 6$ \\
\hline \multirow{10}{*}{ 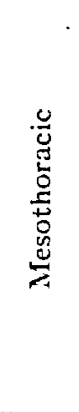 } & I & Rt. & 204 & 120 & 312 & 264 & 114 & 39 & 341256 \\
\hline & & Lft. & 210 & 126 & 312 & 270 & 114 & 42 & 341256 \\
\hline & IV & Rt. & 246 & 120 & 324 & 306 & 120 & 42 & $341(25) 6$ \\
\hline & & Lft. & 234 & 126 & 336 & 312 & 120 & 42 & 341256 \\
\hline & V & Rt. & 240 & 120 & 300 & 276 & 114 & 42 & 341256 \\
\hline & & Ift. & 228 & 120 & 300 & 282 & 114 & 42 & 341256 \\
\hline & VIII & Rt. & 216 & 120 & 294 & 270 & 114 & 33 & 341256 \\
\hline & & Left. & 222 & 120 & 300 & 270 & 114 & 33 & 341256 \\
\hline & IX & Rt. & 210 & 120 & 294 & 276 & 120 & 36 & $341(25) 6$ \\
\hline & & Lft. & 216 & 120 & 300 & 288 & 120 & 36 & $341(25) 6$ \\
\hline \multirow{10}{*}{ 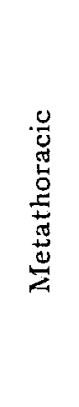 } & I & Rt. & 222 & 126 & 330 & 342 & 114 & 42 & 431256 \\
\hline & & I.ft. & 222 & 126 & 330 & 336 & 114 & 45 & 431256 \\
\hline & IV & R.t. & 252 & 132 & 360 & 390 & 132 & 48 & $431(25) 6$ \\
\hline & & Ift. & 258 & 138 & 348 & 390 & 132 & 48 & 431256 \\
\hline & $\mathrm{V}$ & Rt. & 240 & 126 & 330 & 360 & 120 & 42 & 431256 \\
\hline & & Lft. & 240 & 126 & 330 & 360 & 120 & 42 & 431256 \\
\hline & VIII & Rt. & 216 & 126 & 306 & 336 & 126 & 36 & $431(25) 6$ \\
\hline & & Lft. & 216 & 126 & 306 & 354 & 126 & 36 & $431(25) 6$ \\
\hline & IX & Rt. & 240 & 132 & 336 & 366 & 132 & 39 & $431(25) 6$ \\
\hline & & Lft. & 240 & 132 & 342 & 360 & 132 & 39 & $431(25) 6$ \\
\hline
\end{tabular}


TABle 12. Pseudococcus citri Risso.

\begin{tabular}{|c|c|c|c|c|c|c|c|c|c|}
\hline $\begin{array}{l}\text { Seg- } \\
\text { ment }\end{array}$ & $\begin{array}{l}\text { Spec- } \\
\text { imen }\end{array}$ & Side & Coxa & Tr. & $\begin{array}{l}\mathrm{Fe}- \\
\text { mur }\end{array}$ & Tibia & $\begin{array}{l}\text { Tar- } \\
\text { sus }\end{array}$ & Claws & Formulac \\
\hline \multirow{6}{*}{ 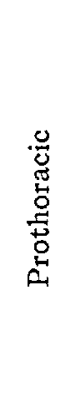 } & I & $\begin{array}{l}\text { Rt. } \\
\text { Lft. }\end{array}$ & $\begin{array}{l}174 \\
174\end{array}$ & $\begin{array}{l}90 \\
90\end{array}$ & $\begin{array}{l}210 \\
104\end{array}$ & $\begin{array}{l}186 \\
180\end{array}$ & $\begin{array}{l}90 \\
90\end{array}$ & $\begin{array}{l}24 \\
24\end{array}$ & $\begin{array}{l}341(25) 6 \\
413(25) 6\end{array}$ \\
\hline & III & Rt. & 198 & 90 & 234 & 210 & 96 & 24 & 341526 \\
\hline & & & & & & & & & \\
\hline & IV & $\begin{array}{l}\text { Rt. } \\
\text { Lft. }\end{array}$ & $\begin{array}{l}180 \\
180\end{array}$ & $\begin{array}{l}90 \\
96\end{array}$ & $\begin{array}{l}216 \\
222\end{array}$ & $\begin{array}{l}192 \\
192\end{array}$ & $\begin{array}{l}84 \\
96\end{array}$ & $\begin{array}{l}24 \\
24\end{array}$ & $\begin{array}{l}341256 \\
341(25) 6\end{array}$ \\
\hline & VI & $\begin{array}{l}\text { Rt. } \\
\text { Lft. }\end{array}$ & $\begin{array}{l}138 \\
138\end{array}$ & $\begin{array}{l}84 \\
84\end{array}$ & $\begin{array}{l}186 \\
186\end{array}$ & $\begin{array}{l}174 \\
174\end{array}$ & $\begin{array}{l}84 \\
84\end{array}$ & $\begin{array}{l}24 \\
24\end{array}$ & $\begin{array}{l}341(25) 6 \\
341(25) 6\end{array}$ \\
\hline & VII & $\begin{array}{l}\text { Rt. } \\
\text { Lft. }\end{array}$ & $\begin{array}{l}174 \\
174\end{array}$ & $\begin{array}{l}90 \\
90\end{array}$ & $\begin{array}{l}210 \\
210\end{array}$ & $\begin{array}{l}192 \\
192\end{array}$ & $\begin{array}{l}90 \\
96\end{array}$ & $\begin{array}{l}24 \\
24\end{array}$ & $\begin{array}{l}341(25) 6 \\
341526\end{array}$ \\
\hline \multirow{5}{*}{ 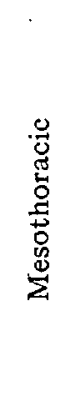 } & I & $\begin{array}{l}\text { Rt. } \\
\text { Lft. }\end{array}$ & $\begin{array}{l}180 \\
186\end{array}$ & $\begin{array}{l}90 \\
96\end{array}$ & 234 & 228 & $\begin{array}{l}102 \\
102\end{array}$ & $\begin{array}{l}27 \\
27\end{array}$ & 341526 \\
\hline & III & $\begin{array}{l}\text { Rt. } \\
\text { Lft. }\end{array}$ & $\begin{array}{l}210 \\
210\end{array}$ & $\begin{array}{l}102 \\
102\end{array}$ & $\begin{array}{l}264 \\
264\end{array}$ & $\begin{array}{l}240 \\
246\end{array}$ & $\begin{array}{l}102 \\
102\end{array}$ & $\begin{array}{l}30 \\
30\end{array}$ & $\begin{array}{l}341(25) 6 \\
341(25) 6\end{array}$ \\
\hline & IV & $\begin{array}{l}\text { Rt. } \\
\text { Lft. }\end{array}$ & $\begin{array}{l}186 \\
174\end{array}$ & $\begin{array}{l}102 \\
102\end{array}$ & $\begin{array}{l}240 \\
240\end{array}$ & $\begin{array}{l}234 \\
228\end{array}$ & $\begin{array}{l}96 \\
96\end{array}$ & $\begin{array}{l}24 \\
24\end{array}$ & $\begin{array}{l}341256 \\
341256\end{array}$ \\
\hline & VI & $\begin{array}{l}\text { Rt. } \\
\text { Lft. }\end{array}$ & $\begin{array}{l}144 \\
156\end{array}$ & $\begin{array}{l}90 \\
90\end{array}$ & $\begin{array}{l}210 \\
210\end{array}$ & $\begin{array}{l}210 \\
210\end{array}$ & $\begin{array}{l}96 \\
96\end{array}$ & $\begin{array}{l}30 \\
30\end{array}$ & $\begin{array}{l}(34) 1526 \\
(34) 1526\end{array}$ \\
\hline & VIJ. & $\begin{array}{l}\text { Rt. } \\
\text { Lft. }\end{array}$ & $\begin{array}{l}180 \\
180\end{array}$ & $\begin{array}{l}96 \\
90\end{array}$ & $\begin{array}{l}240 \\
234\end{array}$ & $\begin{array}{l}228 \\
216\end{array}$ & $\begin{array}{l}96 \\
99\end{array}$ & $\begin{array}{l}27 \\
27\end{array}$ & $\begin{array}{l}341(25) 6 \\
341526\end{array}$ \\
\hline \multirow{5}{*}{ 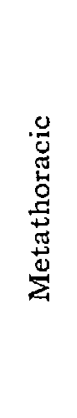 } & I & $\begin{array}{l}\text { Rt. } \\
\text { Lft. }\end{array}$ & $\begin{array}{l}196 \\
196\end{array}$ & $\begin{array}{l}96 \\
96\end{array}$ & $\begin{array}{l}234 \\
240\end{array}$ & $\begin{array}{l}234 \\
248\end{array}$ & $\begin{array}{l}108 \\
108\end{array}$ & $\begin{array}{l}30 \\
30\end{array}$ & $\begin{array}{l}(34) 1526 \\
431526\end{array}$ \\
\hline & III & $\begin{array}{l}\text { Rt. } \\
\text { Lft. }\end{array}$ & $\begin{array}{l}222 \\
216\end{array}$ & $\begin{array}{l}114 \\
108\end{array}$ & $\begin{array}{l}276 \\
276\end{array}$ & $\begin{array}{l}282 \\
282\end{array}$ & $\begin{array}{l}108 \\
108\end{array}$ & $\begin{array}{l}30 \\
33\end{array}$ & $\begin{array}{l}431256 \\
431(25) 6\end{array}$ \\
\hline & IV & $\begin{array}{l}\text { Rt. } \\
\text { Lft. }\end{array}$ & $\begin{array}{l}192 \\
192\end{array}$ & $\begin{array}{l}108 \\
108\end{array}$ & $\begin{array}{l}258 \\
258\end{array}$ & $\begin{array}{l}270 \\
270\end{array}$ & $\begin{array}{l}108 \\
108\end{array}$ & $\begin{array}{l}30 \\
30\end{array}$ & $\begin{array}{l}431(25) 6 \\
431(25) 6\end{array}$ \\
\hline & VI & $\begin{array}{l}\text { Rt. } \\
\text { Lft. }\end{array}$ & $\begin{array}{l}168 \\
168\end{array}$ & $\begin{array}{l}96 \\
96\end{array}$ & $\begin{array}{l}216 \\
228\end{array}$ & $\begin{array}{l}228 \\
240\end{array}$ & $\begin{array}{l}108 \\
108\end{array}$ & $\begin{array}{l}30 \\
30\end{array}$ & $\begin{array}{l}431526 \\
431526\end{array}$ \\
\hline & VII & $\begin{array}{l}\text { Rt. } \\
\text { Lft. }\end{array}$ & $\begin{array}{l}186 \\
194\end{array}$ & $\begin{array}{r}102 \\
92\end{array}$ & $\begin{array}{l}252 \\
252\end{array}$ & $\begin{array}{l}252 \\
246\end{array}$ & $\begin{array}{l}102 \\
102\end{array}$ & $\begin{array}{l}30 \\
30\end{array}$ & $\begin{array}{l}(34) 1(25) 6 \\
341526\end{array}$ \\
\hline
\end{tabular}


TABLE 13. PSEUdococcus longispinus TARG.

\begin{tabular}{|c|c|c|c|c|c|c|c|c|c|}
\hline $\begin{array}{l}\text { Seg- } \\
\text { ment }\end{array}$ & $\begin{array}{l}\text { Spec- } \\
\text { imen }\end{array}$ & Side & Coxa & Tr. & $\begin{array}{l}\mathrm{Fe}- \\
\text { mur }\end{array}$ & Tibia & $\begin{array}{l}\text { Tar- } \\
\text { sus }\end{array}$ & Claws & Formulae \\
\hline \multirow{6}{*}{ 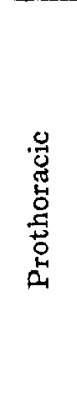 } & VI & $\begin{array}{l}\text { Rt. } \\
\text { lft. }\end{array}$ & $\begin{array}{l}162 \\
180\end{array}$ & $\begin{array}{l}96 \\
96\end{array}$ & $\begin{array}{l}240 \\
240\end{array}$ & $\begin{array}{l}240 \\
240\end{array}$ & $\begin{array}{l}102 \\
102\end{array}$ & $\begin{array}{l}24 \\
24\end{array}$ & $\begin{array}{l}(34) 1526 \\
(34) 1526\end{array}$ \\
\hline & VII & Rt. & 216 & 114 & 270 & 240 & 108 & 24 & 341256 \\
\hline & & Lft. & 216 & 114 & 270 & 240 & 108 & 24 & 341256 \\
\hline & VIII & $\begin{array}{l}\text { Rt. } \\
\text { Lft. }\end{array}$ & $\begin{array}{l}168 \\
180\end{array}$ & $\begin{array}{l}96 \\
96\end{array}$ & $\begin{array}{l}234 \\
234\end{array}$ & $\begin{array}{l}216 \\
216\end{array}$ & $\begin{array}{l}108 \\
108\end{array}$ & $\begin{array}{l}30 \\
27\end{array}$ & $\begin{array}{l}341526 \\
341526\end{array}$ \\
\hline & IX & $\begin{array}{l}\text { Rt. } \\
\text { Lft. }\end{array}$ & $\begin{array}{l}156 \\
156\end{array}$ & $\begin{array}{r}102 \\
96\end{array}$ & $\begin{array}{l}216 \\
216\end{array}$ & $\begin{array}{l}204 \\
204\end{array}$ & $\begin{array}{l}90 \\
96\end{array}$ & $\begin{array}{l}24 \\
24\end{array}$ & $\begin{array}{l}341256 \\
341(25) 6\end{array}$ \\
\hline & $\mathrm{X}$ & $\begin{array}{l}\text { Rt. } \\
\text { Lft. }\end{array}$ & $\begin{array}{l}180 \\
180\end{array}$ & $\begin{array}{l}96 \\
96\end{array}$ & $\begin{array}{l}240 \\
240\end{array}$ & $\begin{array}{l}240 \\
240\end{array}$ & $\begin{array}{l}108 \\
108\end{array}$ & $\begin{array}{l}24 \\
24\end{array}$ & $\begin{array}{l}\text { (43) } 1526 \\
\text { (43)1526 }\end{array}$ \\
\hline \multirow{5}{*}{ 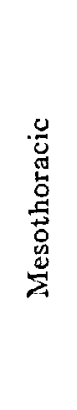 } & VI & $\begin{array}{l}\text { Rt. } \\
\text { Lft. }\end{array}$ & $\begin{array}{l}180 \\
180\end{array}$ & $\begin{array}{l}108 \\
102\end{array}$ & $\begin{array}{l}264 \\
264\end{array}$ & $\begin{array}{l}252 \\
270\end{array}$ & $\begin{array}{l}114 \\
114\end{array}$ & $\begin{array}{l}30 \\
30\end{array}$ & $\begin{array}{l}341526 \\
431526\end{array}$ \\
\hline & VII & $\begin{array}{l}\text { Rt. } \\
\text { Lft. }\end{array}$ & $\begin{array}{l}222 \\
224\end{array}$ & $\begin{array}{l}120 \\
120\end{array}$ & $\begin{array}{l}300 \\
300\end{array}$ & $\begin{array}{l}294 \\
294\end{array}$ & $\begin{array}{l}120 \\
120\end{array}$ & $\begin{array}{l}30 \\
30\end{array}$ & $\begin{array}{l}341(25) 6 \\
341(25) 6\end{array}$ \\
\hline & VIII & $\begin{array}{l}\text { Rt. } \\
\text { Lft. }\end{array}$ & $\begin{array}{l}192 \\
192\end{array}$ & $\begin{array}{l}108 \\
108\end{array}$ & $\begin{array}{l}258 \\
252\end{array}$ & $\begin{array}{l}240 \\
240\end{array}$ & $\begin{array}{l}108 \\
108\end{array}$ & $\begin{array}{l}30 \\
30\end{array}$ & $\begin{array}{l}341(25) 6 \\
341(25) 6\end{array}$ \\
\hline & IX & $\begin{array}{l}\text { Rt. } \\
\text { Lft. }\end{array}$ & $\begin{array}{l}162 \\
162\end{array}$ & $\begin{array}{l}102 \\
102\end{array}$ & $\begin{array}{l}228 \\
228\end{array}$ & $\begin{array}{l}240 \\
234\end{array}$ & $\begin{array}{l}108 \\
108\end{array}$ & $\begin{array}{l}30 \\
30\end{array}$ & $\begin{array}{l}431526 \\
431526\end{array}$ \\
\hline & $X$ & $\begin{array}{l}\text { Rt. } \\
\text { Lft. }\end{array}$ & $\begin{array}{l}192 \\
186\end{array}$ & $\begin{array}{l}108 \\
108\end{array}$ & $\begin{array}{l}264 \\
270\end{array}$ & $\begin{array}{l}264 \\
264\end{array}$ & $\begin{array}{l}108 \\
108\end{array}$ & $\begin{array}{l}30 \\
30\end{array}$ & $\begin{array}{l}(34) 1(25) 6 \\
341(25) 6\end{array}$ \\
\hline \multirow{5}{*}{ 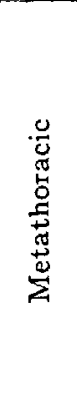 } & VI & $\begin{array}{l}\text { Rt. } \\
\text { Lft. }\end{array}$ & $\begin{array}{l}204 \\
204\end{array}$ & $\begin{array}{l}114 \\
120\end{array}$ & $\begin{array}{l}306 \\
300\end{array}$ & $\begin{array}{l}348 \\
360\end{array}$ & $\begin{array}{l}120 \\
120\end{array}$ & $\begin{array}{l}30 \\
30\end{array}$ & $\begin{array}{l}431526 \\
431(52) 6\end{array}$ \\
\hline & VII & $\begin{array}{l}\text { Rt. } \\
\text { Lft. }\end{array}$ & $\begin{array}{l}240 \\
240\end{array}$ & $\begin{array}{l}132 \\
132\end{array}$ & $\begin{array}{l}312 \\
318\end{array}$ & $\begin{array}{l}\mathbf{3 4 8} \\
354\end{array}$ & $\begin{array}{l}120 \\
120\end{array}$ & $\begin{array}{l}30 \\
30\end{array}$ & $\begin{array}{l}431256 \\
431256\end{array}$ \\
\hline & VIII & $\begin{array}{l}\text { Rt. } \\
\text { Lft. }\end{array}$ & $\begin{array}{l}210 \\
204\end{array}$ & $\begin{array}{l}114 \\
114\end{array}$ & $\begin{array}{l}288 \\
282\end{array}$ & $\begin{array}{l}306 \\
300\end{array}$ & $\begin{array}{l}114 \\
120\end{array}$ & $\begin{array}{l}30 \\
30\end{array}$ & $\begin{array}{l}431(52) 6 \\
431526\end{array}$ \\
\hline & IX & $\begin{array}{l}\text { Rt. } \\
\text { Lft. }\end{array}$ & $\begin{array}{l}180 \\
180\end{array}$ & $\begin{array}{l}108 \\
108\end{array}$ & $\begin{array}{l}252 \\
258\end{array}$ & $\begin{array}{l}294 \\
300\end{array}$ & $\begin{array}{l}120 \\
120\end{array}$ & $\begin{array}{l}30 \\
30\end{array}$ & $\begin{array}{l}431526 \\
431526\end{array}$ \\
\hline & $\mathrm{X}$ & $\begin{array}{l}\text { Rt. } \\
\text { Lft. }\end{array}$ & $\begin{array}{l}204 \\
198\end{array}$ & $\begin{array}{l}120 \\
114\end{array}$ & $\begin{array}{l}300 \\
300\end{array}$ & $\begin{array}{l}318 \\
324\end{array}$ & $\begin{array}{l}114 \\
114\end{array}$ & $\begin{array}{l}30 \\
30\end{array}$ & $\begin{array}{l}431256 \\
431(25) 6\end{array}$ \\
\hline
\end{tabular}


TABLE 14. PSEUdococcus obscurus Essig.

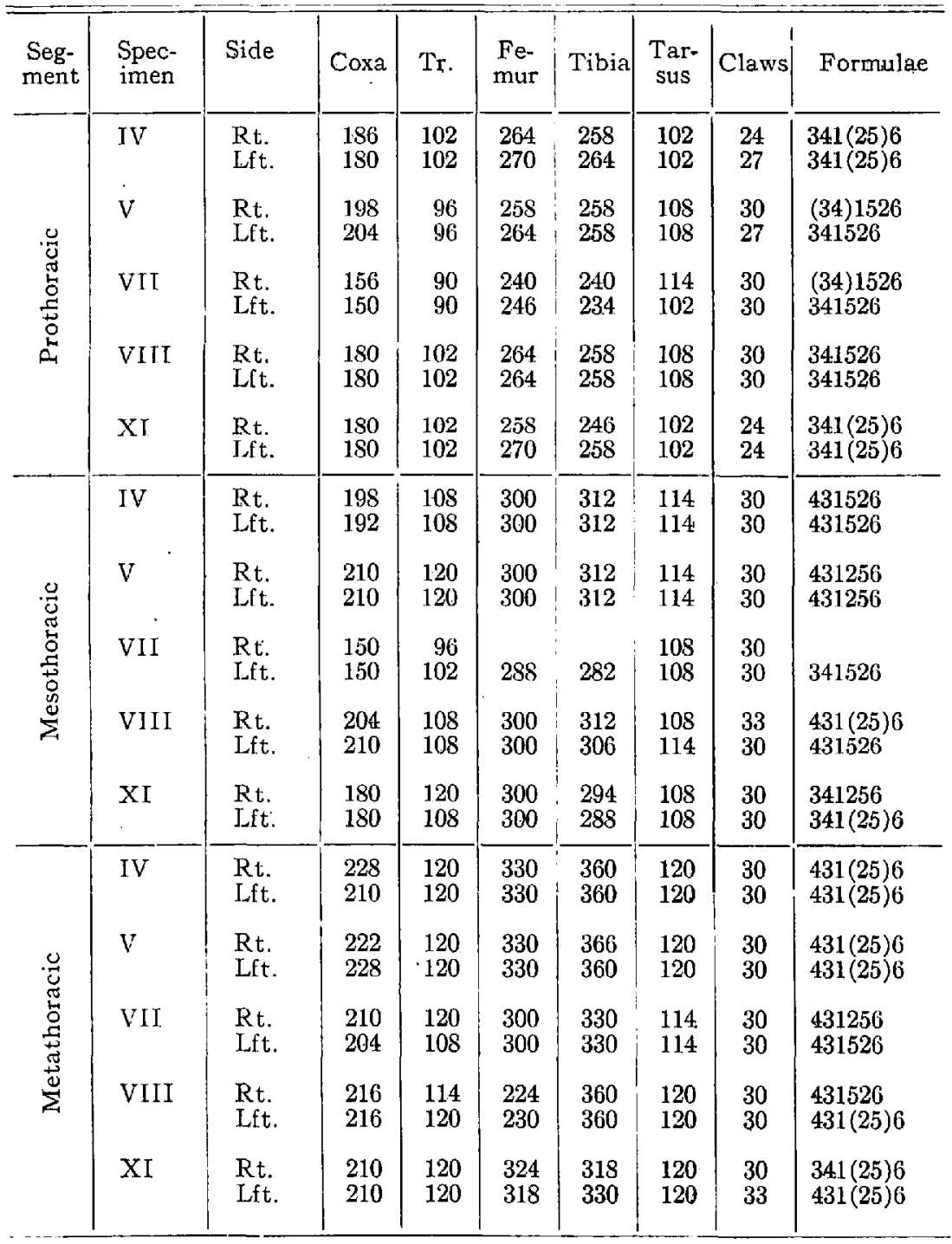


TABLE 15. Pseudococcus agrifoliae Essig.

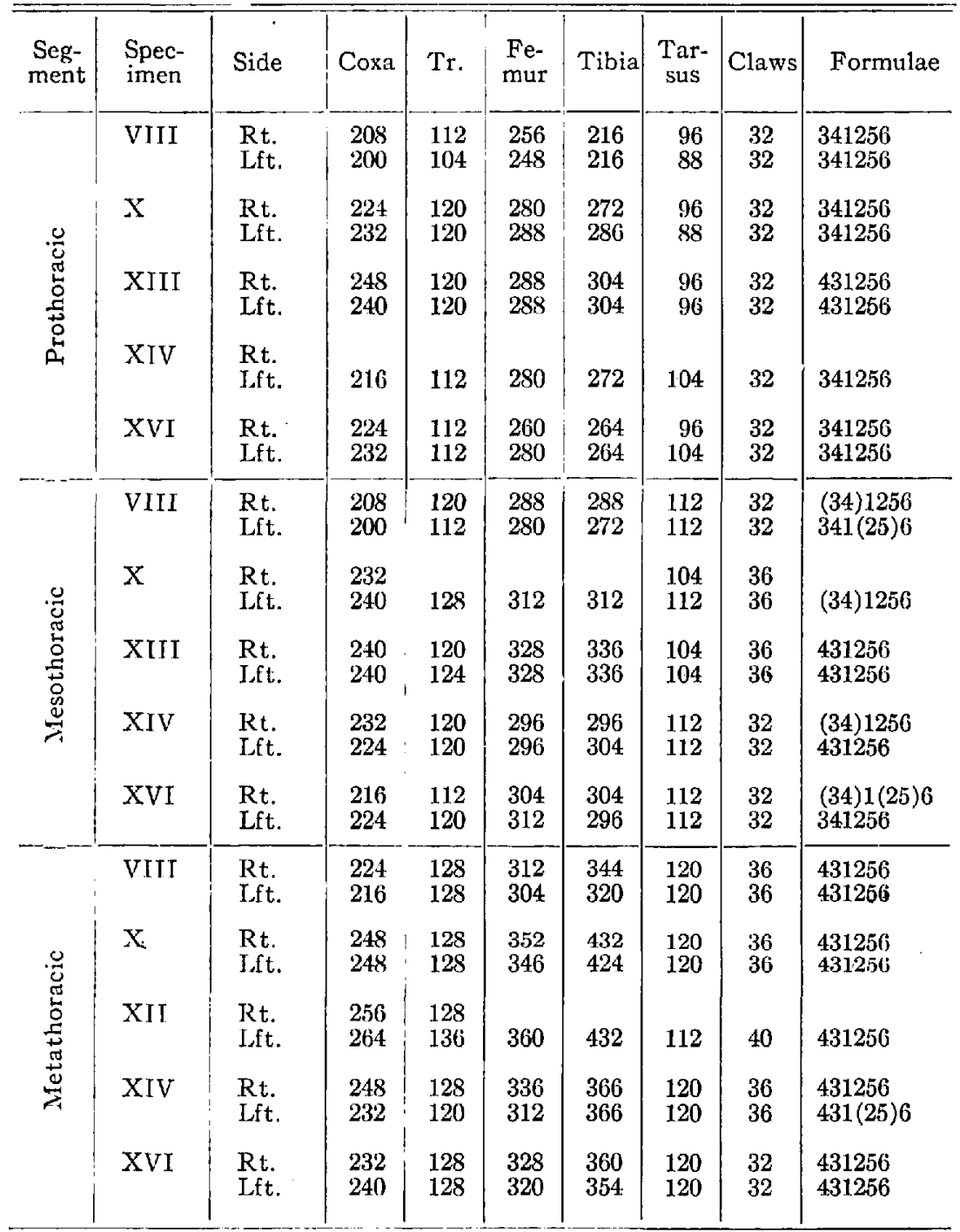

MaŔginal Wax Filaments. The number, length and stoutness of the marginal wax filaments is a character often used. The length of the filaments compared to the length of the body and to each other is the form most used. The favorite character for separating longispinus Targ. and citri Risso is the extreme length of the caudal filaments in the former. 
This character refers to specimens before treatment with caustic potash. Only a slight study has been made of this character because living specimens of three of the five species studied were not available. A difference however has been noted between citri Risso and longispinus Targ., in the former the appendages are robust and covered with granules of wax, in the latter the appendages are slender and do not have granules upon them, being more linear and wire like. The objection to the use of this character in the determination of species is the ease with which the filaments are removed and the danger that the specimens consequently would not be in a natural condition. Also in recently moulted specimens the normal waxy covering would not be formed. The length of the caudal filaments in longispinus Targ. is evidently a good character if the specimens can be examined in a normal condition.

ANAL LoBEs.--The anal lobes are frequently mentioned as being faintly indicated, normal or prominent. Also the presence of one or more setae is occasionally used.

The observations made upon the segmentation of specimens would apply very largely to a discussion of the anal lobes. As a specimen becomes older and more distended with eggs the anal lobes become less prominent. Consequently in the use of this character the age of the individual would have to be carefully considered. In the setae of the anal lobes there is evidently a good character. (Tables 1-5). Upon each anal lobe there are generally several setae. One of these, however, is much longer and more robust than the others and is usually situated at the caudal extremity of the lobe. This is the seta referred to in the tables and discussion. The variation in the length of this seta is considerable, but the limits of variation are so great that this does not lessen the value of the character. The comparison of the length of this seta to the length of the setae of the anal. ring is probably the most useful way of expressing the character.

Setae of the ANAL Ring.-The number of setae on the anal ring is a generic character and although often given in descriptions is of no importance as a specific character. Their length compared to some other part of the insect is seldom mentioned.

However, the length of these setae is a very promising specific character. The variation is considerable but not so 
great as to nullify the usefulness of the character. To deter. mine the distal termination of the setae is sometimes difficult, but it can be done with considerable accuracy. Another difficulty is that the distal end of the setae have a tendency to curl, but in spite of this and the above mentioned factor the length of a seta can usually be quite accurately measured. As stated above the comparison of the length of the setae of the anal ring with the setae of the anal lobes is probably the most available way to use this character.

In agrifoliae Essig considerable variation is found and the character is hardly satisfactory, The setae of this species are especially difficult to measure because they are not robust.

DERMIS.- The presence of setae and groups of spinnerets upon the dermis is a character commonly noted. This is usually not given enough in detail to be of assistance in the separation of species.

EGG-SAC.-Considerable use is made of the egg laying habits of the species. Whether the egg-sac is of a fibrous nature or is a mealy secretion is often stated. Also its presence or absence is noted, depending whether the species is ovoviviparous or oviparous.

If a study of specimens of Pseudococcus can be made with the insects in a natural condition, the manner of oviposition is a good specific character. Whether a species is ovoviviparous or oviparous is not always a sharply separated condition, for an individual may give birth to living young and also deposit eggs. However, the formation of an egg sac is an indication that a species is oviparous and the formation of this egg-sac is constant for the species. In a normally ovoviviparous form a mealy secretion which is distinctly of a fibrous nature is made upon the plant. Crawii Coq. and longispinus Targ. do not form a distinct egg-sac but form a mealy secretion upon the plant. Citri Risso and obscurus Essig form an egg-sac of considerable size. Agrifoliae Essig forms an egg-sac but not so extensive a one as the last two named species.

The writer is working on a monograph of the North American species of this genus. He would be glad to exchange specimens of Coccidae for species of Pseudococcus not now in his collection. Correspondence to this end is invited. 